\title{
Serviços de apoio à saúde mental do estudante de Medicina: uma revisão sistemática
}

\author{
Mental health support services for medical students: a systematic review
}

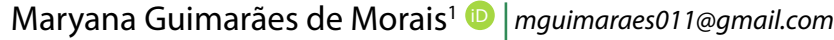 \\ Isabella Morais Arantes de Oliveira e Silva' (D) bebellaarantes@gmail.com \\ Estela Ribeiro Versiani' (1) estela.versiani@gmail.com \\ Claudia Cardoso Gomes da Silva' (D) claudiacardoso.cpeq@gmail.com \\ Ana Socorro de Moura' (1) ana10escs@gmail.com
}

\section{RESUMO}

Introduç̧ão: Considerando a alta prevalência de problemas de saúde mental entre estudantes de Medicina, as instituições que formam profissionais médicos têm o compromisso ético de se preocupar com a promoção da saúde mental de seu corpo discente, oferecendo serviços de apoio e desenvolvendo estratégias de prevenção.

Objetivo: Esta revisão tem como objetivo identificar publicações científicas sobre serviços de assistência oferecidos aos estudantes de Medicina nas instituições de ensino superior do Brasil, bem como informações sobre os profissionais que os compõem, o público-alvo atendido e os tipos de intervenção mais utilizados.

Método: Trata-se de revisão sistemática de literatura, orientada pelas diretrizes do Preferred Reporting Items for Systematic Reviews and MetaAnalysis (PRISMA). Foi realizada busca por estudos nas bases de dados SciELO, PubMed/Medline, Lilacs, ERIC, The Cochrane Library e Catálogo de Teses e Dissertações da Capes, sem delimitação de tempo, publicados até agosto de 2020. Em seguida, duas revisoras, de forma independente, selecionaram os estudos e extraíram os dados pertinentes para a construção desta revisão.

Resultado: Foram incluídos 16 estudos. Os serviços de apoio identificados atuam por meio de estratégias diversas com o objetivo comum de promoção da saúde mental do estudante. As intervenções mais encontradas nesses serviços são atendimento psicoterápico breve, atendimento psiquiátrico, orientação psicopedagógica e programas de mentoring. A maioria dos serviços foi implementada para atender estudantes de Medicina, e alguns ampliaram seu alcance a discentes de outros cursos de graduação. Em relação aos profissionais que compõem esses serviços, encontramos equipes multiprofissionais que variam em número e categorias profissionais envolvidas.

Conclusão: Os serviços de apoio destinados ao estudante de Medicina no Brasil apresentam diferenças tanto quanto à forma de atuação dentro das instituições como quanto aos profissionais envolvidos. Verificou-se que as publicações sobre esses serviços são escassas quando comparadas ao número de instituições que oferecem o curso de graduação em Medicina no Brasil. A fim de ampliar e consolidar ações voltadas para a promoção da saúde mental do estudante de Medicina dentro das instituições de ensino superior brasileiras, mais pesquisas sobre essa temática são necessárias.

Palavras-chave: Saúde Mental; Estudantes de Medicina; Saúde do Estudante; Serviços de Saúde Mental.

\section{ABSTRACT}

Introduction: Considering the high prevalence of mental health problems among medical students, medical schools should be ethically committed to promote student's mental health, offering health services and prevention strategies.

Objective: The objective of this systematic review is to identify scientific publications on mental health services offered by Brazilian universities to medical students, as well as the professionals involved and the types of interventions most often offered.

Method: Systematic literature review, following the guidelines of the Preferred Reporting Items for Systematic Reviews and Meta-analysis (PRISMA). The Scielo, PubMed/Medline, Lilacs, ERIC, The Cochrane Library and CAPES databases were searched for studies published until August 2020, as well as theses and dissertations. The studies were screened, selected, analyzed and relevant data were extracted by two independent reviewers.

Results: 16 studies were included. The identified mental health services resort to different strategies towards a common goal of promoting medical students mental health. The interventions most frequently found in these services are brief psychotherapy, psychiatric care, psychological-educational guidance and mentoring programs. Most services were created to attend medical students and some of these have been extended to other undergraduate students. In the analyzed services, there is a predominance of multidisciplinary teams, which differ regarding the number and categories of professionals involved.

Conclusion: Mental health support services for medical students in Brazil differ both in terms of how they work within the institutions and in terms of the involved professionals. It was observed that publications on these services are scarce when compared to the number of institutions that offer the undergraduate medical course in Brazil. Aiming to expand and consolidate actions aimed at promoting medical students' mental health in Brazilian higher education institutions, further research on this topic is required.

Keywords: Mental Health; Medical Students; Student Health; Mental Health Services.

${ }^{1}$ Escola Superior de Ciências da Saúde, Brasília, Distrito Federal, Brasil. 


\section{INTRODUÇÃO}

Elevada taxa de transtornos mentais em estudantes de Medicina tem sido relacionada a uma série de fatores inerentes tanto ao contexto universitário como ao próprio indivíduo, que, ao ingressar na graduação, deve lidar com questões associadas à adolescência, à transição para a universidade, à adaptação a diferentes métodos de aprendizagem e ao estabelecimento de novas relações interpessoais ${ }^{1-3}$.

Em estudo de revisão sistemática com metanálise de 2017, verificou-se a prevalência de diversos problemas de saúde mental em estudantes de Medicina no Brasil, incluindo depressão, burnout, uso problemático de álcool e ansiedade. Dentre esses, o mais prevalente, assim como na população geral, foi a ansiedade, indicando que a maior parte dos acadêmicos de Medicina no país manifesta tendência de apresentar sintomas de ansiedade. A análise mostrou ainda que os sinais de depressão apresentados pelos estudantes, quando estratificados por sua severidade, são predominantemente leves².

Em pesquisa qualitativa transversal, realizada por Tenório et al. ${ }^{4}$ em 2016, procurou-se avaliar a saúde mental de 78 discentes de duas escolas médicas com diferentes modelos de ensino e aprendizagem, sendo uma delas de abordagem tradicional e outra estruturada na aprendizagem baseada em problemas (ABP). Por meio de questionário semiestruturado e de grupos focais constituídos pelos estudantes, constatou-se que o processo educacional foi associado a sofrimento psíquico por ambos os grupos, que referiram motivações, fontes de estresse relacionadas ao curso e fatores de alívio semelhantes.

Nesse contexto, em que a formação médica está muitas vezes associada a transtornos psíquicos e emocionais, as instituições que formam profissionais médicos têm 0 compromisso ético de se preocupar com a saúde mental de seus estudantes, oferecendo estratégias de prevenção e cuidados nessa área.

No Brasil, a preocupação das instituições de ensino superior com a saúde mental de seus estudantes adquiriu maior relevância na segunda metade do século XX, com a implantação de serviços que ofereciam assistência psicológica ao estudante universitário. O primeiro serviço com essa finalidade foi criado em 1957 na Universidade Federal de Pernambuco e teve os graduandos de Medicina como primeiros contemplados. Nesse mesmo contexto, nos anos 1960, as universidades federais do Rio Grande do Sul, de Minas Gerais e do Rio de Janeiro também implementaram serviços de saúde mental destinados aos discentes. Desde então, diversos projetos com função assistencial voltados para a promoção da saúde mental dos estudantes têm sido implementados em centros de ensino superior do Brasil ${ }^{5,6}$.

Com o intuito de garantir o sucesso da assistência psicológica e psiquiátrica dentro do ambiente acadêmico, considera-se que o sigilo, a independência e o princípio de flexibilidade são as principais características que os serviços assistenciais destinados aos universitários devem apresentar. A confidencialidade das informações compartilhadas no atendimento, a independência do serviço em relação aos departamentos de ensino, com uma equipe de assistência desvinculada do corpo docente, e a flexibilidade do tipo de atendimento oferecido de acordo com as circunstâncias e a identidade de cada indivíduo contribuem para que os serviços dessa natureza cumpram com excelência e ética a função assistencial ${ }^{7}$.

Em 2016, no I Fórum Paulista de Serviços de Apoio aos Estudantes de Medicina, foi elaborada "A carta de Marília". Nesse documento, construído coletivamente por representantes de serviços de apoio discente, docentes e estudantes, enfatizouse a responsabilidade das instituições de ensino na promoção e recuperação da saúde mental dos universitários. Dentre as ações que podem contribuir para esse fim, elencaramse atuações que vão desde o atendimento individualizado por profissionais qualificados até a promoção de atividades culturais que extrapolam o ambiente acadêmico ${ }^{8}$.

Contudo, desafios quanto à implantação e ampliação de serviços de assistência ao discente de Medicina, já existentes no período de introdução dessas abordagens, permanecem. Verifica-se que as demandas atuais das escolas médicas do país estão vinculadas à adequação dos atendimentos ao modelo individualizado e à aplicação de novas estratégias de apoio, como o incentivo ao desenvolvimento de atividades extracurriculares ${ }^{9}$.

A fim de contribuir para a discussão a respeito dos serviços de apoio ao estudante de Medicina em instituições brasileiras, torna-se importante mapear e conhecer as publicações existentes sobre esse tipo de serviço, assim como compreender sua forma de funcionamento. Espera-se com isso oferecer subsídios para o aperfeiçoamento das ações de promoção da saúde mental oferecidas pelos serviços de centros de ensino médico e, em última instância, colaborar para a qualidade do atendimento prestado ao estudante de Medicina.

Nesse sentido, este estudo tem como objetivo identificar publicações científicas sobre os serviços de assistência oferecidos aos estudantes de Medicina nas instituições de ensino superior do Brasil, bem como informações sobre os profissionais que os compõem, o público-alvo atendido e os tipos de intervenção mais utilizados nesses serviços.

\section{Questões da revisão}

As seguintes perguntas conduziram a pesquisa:

- O que já foi publicado sobre os serviços de apoio à saúde mental disponibilizados aos estudantes 
nas instituições de ensino superior que oferecem o curso de Medicina no Brasil?

- Quais são as estratégias adotadas pelos serviços de apoio no sentido de promoção da saúde mental?

- Quais profissionais são responsáveis pela oferta desses serviços de apoio à saúde mental do estudante?

\section{MÉTODO}

\section{Tipo de estudo}

Trata-se de uma revisão sistemática de literatura orientada pelas diretrizes do Preferred Reporting Items for Systematic Reviews and Meta-Analysis (PRISMA) e realizada para identificar estudos que descrevem serviços de apoio à saúde mental de estudantes do curso de Medicina, no contexto das instituições de ensino superior no Brasil.

\section{Critérios de inclusão e exclusão}

Incluíram-se na revisão todos os estudos encontrados em português, inglês ou espanhol que abordavam a existência de serviços de apoio à saúde mental do estudante de Medicina nas instituições de ensino superior do Brasil, sem delimitação de tempo, mas que fossem publicados até agosto de 2020, momento da última busca.

Excluíram-se estudos que tratavam de intervenções voltadas à promoção da saúde mental do estudante de Medicina que não haviam sido promovidas por um serviço estruturado para essa finalidade e estudos cujo acesso ao texto completo não foi possível.

\section{Bases de dados}

Uma pesquisa sistemática foi conduzida a partir de artigos e dissertações publicados nas seguintes bases de dados: PubMed/Medline, Lilacs, The Cochrane Library, SciELO, ERIC e Catálogo de Teses e Dissertações da Coordenação de Aperfeiçoamento de Pessoal de Nível Superior (Capes).

\section{Estratégia de busca}

A estratégia de busca foi definida a partir de uma pesquisa avançada nessas bases, com o intuito de incluir a maior quantidade de estudos relevantes. Os descritores utilizados como referência foram "serviços de apoio", "saúde mental" e "estudantes de medicina", bem como seus correspondentes em inglês support services, mental health e medical students. As estratégias de busca foram adaptadas para os diferentes bancos de dados e são descritas no Quadro 1.

\section{Seleção dos estudos e extração de dados}

Todas as referências encontradas pelas buscas foram organizadas com auxílio do Software Mendeley ${ }^{10}$. Em seguida, utilizou-se a Plataforma Covidence para Revisão Sistemática, da Colaboração Cochrane ${ }^{11}$, como ferramenta para a seleção e extração de dados dos estudos.

A seleção dos estudos foi realizada, de forma independente, por duas revisoras devidamente treinadas. A Plataforma Covidence disponibiliza uma interface para cada revisor e depois indica quais foram os estudos que apresentaram divergências na análise, para que estas sejam resolvidas por um terceiro revisor. Inicialmente, analisaram-se o título e o resumo dos textos selecionados. As divergências sobre a inclusão ou exclusão de determinado estudo foram analisadas por uma terceira revisora. Após essa etapa, partiuse para a avaliação dos textos completos e definição das espécies que comporiam a revisão. Novamente, para estudos em que houve desacordo entre as duas revisoras principais, a terceira revisora resolveu os conflitos.

Os dados foram extraídos dos estudos incluídos utilizando tabelas de extração de dados projetadas para atender aos objetivos desta revisão. As características dos estudos foram retiradas dos resumos e dos textos completos dos trabalhos. Duas revisoras extraíram os dados independentemente, e, nos casos de discordância, os dados foram analisados por uma terceira revisora.

Quadro 1. Estratégias de busca.

\begin{tabular}{|c|c|c|}
\hline $\begin{array}{c}\text { Bases de } \\
\text { dados }\end{array}$ & Estratégias de busca & $\begin{array}{c}\text { Total de estudos } \\
\text { encontrados }\end{array}$ \\
\hline $\begin{array}{l}\text { PubMed/ } \\
\text { Medline }\end{array}$ & $\begin{array}{c}\text { ((((support services) OR } \\
\text { (psychological support)) } \\
\text { AND (mental health)) } \\
\text { AND (medical students)) } \\
\text { AND (brazil) } \\
\end{array}$ & 20 estudos \\
\hline Lilacs & $\begin{array}{c}\text { (tw:(serviços de apoio )) } \\
\text { OR (tw:(apoio psicológico)) } \\
\text { OR (tw:(apoio )) AND } \\
\text { (tw:(mental health )) AND } \\
\text { (tw:(medical students )) }\end{array}$ & 31 estudos \\
\hline $\begin{array}{c}\text { The Cochrane } \\
\text { Library }\end{array}$ & $\begin{array}{c}\text { \#1 (support service) OR } \\
\text { (psychological support) \#2 } \\
\text { (mental health) \#3 (medical } \\
\text { students) \#4 (brazil) \#5 (\#1 } \\
\text { AND \#2 AND \#3 AND \#4 } \\
\text { AND \#5) }\end{array}$ & 63 estudos \\
\hline ERIC & $\begin{array}{l}\text { "support service" } \\
\text { "psychological support" } \\
\text { "mental health" "medical } \\
\text { students"“brazil" }\end{array}$ & 6 artigos \\
\hline SciELO & $\begin{array}{l}\text { (ab:(* serviços de apoio)) } \\
\text { OR (apoio) OR (apoio } \\
\text { psicológico) AND (saúde } \\
\text { mental) AND (estudantes } \\
\text { de medicina) }\end{array}$ & 4 artigos \\
\hline $\begin{array}{l}\text { Catálogo } \\
\text { de Teses e } \\
\text { Dissertações } \\
\text { da Capes }\end{array}$ & $\begin{array}{c}\text { serviços de apoio OR apoio } \\
\text { OR apoio psicológico } \\
\text { AND saúde mental AND } \\
\text { estudantes de medicina }\end{array}$ & 47 artigos \\
\hline
\end{tabular}


Figura 1. Diagrama de fluxo PRISMA.

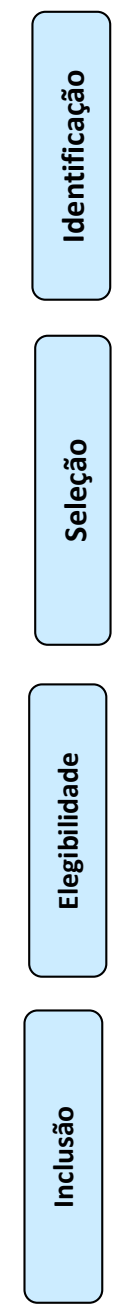

Fonte: Elaborado pelas autoras a partir do PRISMA 2009 Flow Diagram.

Encontraram-se 171 estudos por meio das buscas nas bases de dados. Além desses, triaram-se outros 20 artigos, identificados nas referências de estudos selecionados.

Do total de 191 estudos reunidos, 57 foram excluídos pela própria Plataforma Covidence por serem duplicatas, restando 134 estudos para serem triados a partir do título e do resumo. Os textos completos de 61 estudos foram avaliados quanto à elegibilidade, e, após a exclusão de 45 deles por não cumprirem os critérios de inclusão, restaram os 16 estudos incluídos (Figura 1).

\section{RESULTADOS E DISCUSSÃO}

\section{Características dos estudos incluídos}

Com base na pesquisa bibliográfica, selecionaram-se 16 estudos para a construção desta revisão. Destes, 13 são artigos publicados em periódicos e três são dissertações apresentadas a programas de pós-graduação de instituições de ensino superior $^{12-14}$. Em relação ao desenho metodológico desses estudos, o tipo predominante foi a pesquisa documental de prontuários e de fichas de inscrição disponibilizados nos serviços de apoio à saúde mental. Outros desenhos presentes são relato de experiência ${ }^{15,16}$ e descrição histórica ${ }^{17,18}$ além de pesquisa qualitativa com a aplicação de questionários ${ }^{19}$ e com a análise fenomenológica de depoimentos ${ }^{13,20}$.

Todos os estudos selecionados foram realizados no Brasil, dos quais 14 envolvem instituições de ensino superior da Região Sudeste. Apenas dois estudos foram desenvolvidos em outras localidades: um em instituição da Região Norte ${ }^{16}$ e outro em um centro de ensino superior da Região Sul ${ }^{21}$. Constata-se, assim, uma concentração de publicações sobre os serviços de apoio à saúde mental destinados ao estudante de Medicina nos centros de ensino da Região Sudeste. Além disso, chama a atenção a existência de poucos estudos sobre esse tema, especialmente quando se considera o número de instituições que oferecem o curso de graduação em Medicina no Brasil 287 instituições de ensino superior, públicas e privadas ${ }^{22}$.

Os dados revelam que 13 dos estudos realizados ocorreram em instituições públicas de ensino superior e que apenas três ${ }^{21,23,24}$ foram realizados em centros privados, o que, possivelmente, pode ser efeito de uma política de 
maior incentivo a pesquisas nas instituições públicas. Estudos apontam que o setor público está mais estruturado para a pesquisa do que o setor privado por causa das políticas educacionais adotadas historicamente no país. Embora tenha havido um maior incentivo às instituições privadas na última década, esse estímulo não foi destinado à pesquisa, mas à formação profissional| ${ }^{25}$.

Alguns serviços de apoio à saúde mental são descritos em mais de um estudo, como é o caso do Grupo de Assistência Psicológica ao Aluno da Faculdade de Medicina da Universidade de São Paulo (Grapal/FMUSP), abordado em dois estudos selecionados ${ }^{18,26}$; do Serviço de Assistência Psicológica e Psiquiátrica ao Estudante da Universidade Estadual de Campinas (Sappe/Unicamp), presente nos estudos de Campos $^{12}$ e Oliveira ${ }^{14}$; e do Centro de Apoio Educacional e Psicológico da Faculdade de Medicina de Ribeirão Preto (Caep/ FMRP), também descrito em dois estudos ${ }^{15,17}$. Essa informação corrobora a constatação de que os estudos sobre os serviços de apoio ao estudante de Medicina estão concentrados em instituições de ensino públicas da Região Sudeste.

Por último, é válido ressaltar que os estudos incluídos nesta revisão analisaram principalmente o processo de implantação do serviço de apoio à saúde mental, as características do serviço oferecido, os traços sociodemográficos e clínicos do público atendido, e os principais motivos de busca por atendimento nesses serviços.

\section{Criação dos serviços}

Cada instituição de ensino, ao criar um serviço de apoio à saúde mental de seus estudantes, procurou atender às demandas próprias de seu contexto acadêmico. $\mathrm{O}$ Espaço de Atenção Psicossocial (Epsico) da Universidade do Estado do Amazonas (UEA) ${ }^{16}$ e o Projeto de Assistência Psicológica da Pontifícia Universidade Católica de Minas Gerais (APP/PUC Minas ${ }^{23}$ são exemplos de casos em que a ideia de oferecer estratégias para promoção da saúde mental partiu da gestão da unidade acadêmica, diante de situações de sofrimento psíquico dos estudantes.

Nos dois casos, o processo foi semelhante: diante de um número crescente de estudantes com demandas acadêmicas e psicossociais relacionadas ao ingresso na faculdade e à adaptação a esse cenário, uma comissão de professores e gestores foi instituída a fim de mobilizar recursos e estratégias para a implementação de um serviço voltado para o discente.

Além disso, é válido ressaltar que a atuação de docentes ligados às disciplinas de psiquiatria e psicologia médica foi importante para a criação dos serviços de apoio em muitas instituições. O Caep da FMRP ${ }^{17}$ e o Grapal da FMUSP ${ }^{18}$ foram projetados e fomentados por professores que conviviam de perto com os estudantes de Medicina e compreendiam a necessidade de ofertar assistência psicológica a esse público.

Diante desses exemplos, observa-se a importância da articulação entre a comunidade acadêmica e os gestores das instituições de ensino médico na implantação dos serviços de apoio à saúde mental de seus estudantes.

\section{Tipos de serviço e intervenção oferecidos}

Os serviços de apoio à saúde mental descritos nos estudos selecionados estão estruturados, em sua maioria, para oferecer um primeiro acolhimento, como atendimento psicológico individual por um curto período de tempo, em casos de sofrimento psíquico relacionado a questões pessoais e/ou acadêmicas.

A maioria dos serviços dispõe de horários de funcionamento preestabelecidos, e a procura por atendimento acontece por demanda espontânea, sem a necessidade de agendamento prévio. Apenas no Epsico da UEA ${ }^{16}$ e no Núcleo de Apoio ao Estudante da Faculdade de Medicina da Universidade Federal de Minas Gerais (Napem/UFMG) ${ }^{27}$, o atendimento ocorre, respectivamente, com agendamento por mensagens de texto ou pelo preenchimento de uma ficha de inscrição, na qual o estudante informa previamente o motivo da consulta.

Após a procura por atendimento, o estudante geralmente passa por uma triagem psicológica, na qual é levantada sua principal queixa ou motivo de busca por atendimento e definida a intervenção mais adequada. No Programa de Atenção em Saúde Mental (Proasme), implementado pela Universidade Federal do Rio de Janeiro (UFRJ), por exemplo, esse contato inicial é denominado "Recepção Ampliada" e visa à realização de um diagnóstico situacional a partir da elaboração das razões que levaram o estudante a se dirigir ao serviço ${ }^{28}$.

Foi observado que as intervenções psicológicas oferecidas são de modalidades diversas, com predomínio da psicoterapia breve de orientação psicanalítica. Esse tipo de intervenção é caracterizado por um número reduzido de atendimentos - de seis a dez sessões-, com cada sessão durando cerca de 50 minutos. O principal objetivo da psicoterapia breve é propiciar ao paciente a aquisição de insight, isto é, o conhecimento da própria realidade psíquica por meio da interpretação dos relatos expostos no atendimento e de seus contextos vivenciais ${ }^{16}$.

Na maioria dos estudos, é ressaltado ainda que, em casos de transtornos mentais mais graves ou que necessitam de abordagens de longo prazo, o estudante é encaminhado a atendimentos psicológicos e/ou psiquiátricos desvinculados da instituição de ensino.

Nesse sentido, seria bastante apropriado que os serviços de apoio estivessem associados à Rede de Atenção 
Psicossocial (Raps) do Sistema Único de Saúde (SUS) ou estabelecessem parcerias com ela, a fim de garantir a assistência adequada principalmente aos estudantes contemplados por ações afirmativas e/ou em situações de vulnerabilidade socioeconômica, que não podem arcar com os custos de um atendimento particular.

Outros exemplos de modalidades de intervenção são a orientação psicopedagógica ou pedagógica realizadas no Grapal da FMUSP ${ }^{18,26}$ e o pronto atendimento psicológico (PAP) oferecido no Sappe da Unicamp ${ }^{12,14}$.

Alguns serviços de apoio contam também com atendimento psiquiátrico. Nesse caso, o estudante pode solicitar esse tipo de atendimento de forma exclusiva ou associado ao atendimento psicológico, como ocorre no Serviço de Psicoterapia Analítica do Ambulatório de Clínica Psiquiátrica do Hospital das Clínicas de Ribeirão Preto ${ }^{29}$. Somente na Retaguarda Emocional para o Aluno de Medicina (Repam), serviço da Faculdade de Ciências Médicas da Santa Casa de São Paulo, relata-se a prescrição de medicações pelo psiquiatra ${ }^{24}$. No entanto, são poucos os serviços que oferecem essa modalidade de atendimento, provavelmente por conta da ausência de um médico psiquiatra atuando nesses projetos.

A tutoria ou mentoring é outro tipo de intervenção oferecido, como ocorre no Programa de Mentoring da UFMG ${ }^{20} \mathrm{e}$ no Mentoring Program da FMUSP ${ }^{19}$. Segundo estudo publicado por Leão et al. ${ }^{19}$ em 2011, essa forma de assistência tem como objetivo oferecer um tutor (mentor) para acompanhar os estudantes ao longo da sua formação e promover a troca de experiências entre docentes e discentes. Nesse sentido, os graduandos são divididos em grupos, que podem variar de 12 a 14 pessoas, com um tutor/docente ficando responsável por cada grupo. São desenvolvidas reuniões regulares para a discussão e orientação de aspectos relacionados à carreira médica e de questões pertinentes à vida pessoal dos estudantes.

Muitos serviços atuam também no desenvolvimento de pesquisas e projetos que visam promover a saúde mental dentro do ambiente acadêmico e oferecer subsídios para o aprimoramento do ensino dentro da instituição. Como exemplo dessa atuação, podemos elencar programas que facilitam a integração do estudante ao meio universitário, desenvolvidos pelo Caep da FMRP15,17, e a produção científica de estudos sobre as necessidades da população estudantil. Ainda nesse sentido, o Caep presta assessoria à Comissão de Graduação da instituição, com o intuito de sugerir e implementar propostas pedagógicas que associem a formação médica ao bem-estar dos graduandos ${ }^{15,17}$.

Diante dos dados analisados, é possível perceber que os serviços de apoio ao estudante descritos nos estudos incluídos nesta revisão apresentam o objetivo comum de promover a saúde mental dos discentes de Medicina. Divergem, entretanto, quanto às modalidades de intervenção oferecidas - que vão desde $o$ atendimento individual até atuações em outros setores da instituição -, demonstrando não haver uma única maneira de abordar de forma efetiva a questão da saúde mental nas instituições de ensino superior. O Quadro 2 apresenta os principais dados coletados dos estudos incluídos nesta revisão.

Quadro 2. Principais dados coletados.

\begin{tabular}{|c|c|c|c|c|c|}
\hline $\begin{array}{l}\text { Autor(es)/ano } \\
\text { de publicação }\end{array}$ & $\begin{array}{c}\text { Formato/ } \\
\text { desenho } \\
\text { metodológico }\end{array}$ & $\begin{array}{l}\text { Nome do serviço/ano de } \\
\text { criação }\end{array}$ & Intervenções oferecidas & Público-alvo & $\begin{array}{c}\text { Profissionais } \\
\text { que compõem o } \\
\text { serviço }\end{array}$ \\
\hline $\begin{array}{l}\text { Fernandez } \\
\text { e Rodrigues } \\
\text { (1993) }\end{array}$ & $\begin{array}{l}\text { Artigo/ } \\
\text { pesquisa } \\
\text { documental }\end{array}$ & $\begin{array}{l}\text { Serviço de Psicoterapia } \\
\text { Analítica do Ambulatório } \\
\text { de Clínica Psiquiátrica do } \\
\text { Hospital das Clínicas de } \\
\text { Ribeirão Preto - o ano de } \\
\text { implantação do serviço não } \\
\text { foi informado. O atendimento } \\
\text { aos estudantes de Medicina } \\
\text { foi iniciado em } 1983 \text {. }\end{array}$ & $\begin{array}{l}\text { Psicoterapia analítica e } \\
\text { atendimento psiquiátrico }\end{array}$ & $\begin{array}{l}\text { Estudantes dos cursos } \\
\text { ofertados por instituições de } \\
\text { ensino superior de São Paulo, } \\
\text { entre as quais se destaca a } \\
\text { Universidade de São Paulo } \\
\text { (USP). As demais não foram } \\
\text { apresentadas. A maior procura } \\
\text { refere-se aos graduandos de } \\
\text { Medicina e Psicologia. }\end{array}$ & $\begin{array}{l}\text { Médicos } \\
\text { residentes de } \\
\text { psiquiatria, } \\
\text { aprimorandos } \\
\text { de psicologia } \\
\text { e médicos } \\
\text { assistentes da } \\
\text { instituição. }\end{array}$ \\
\hline $\begin{array}{l}\text { Cianflone et al. } \\
(2002)\end{array}$ & $\begin{array}{l}\text { Artigo/ } \\
\text { descrição } \\
\text { histórica }\end{array}$ & $\begin{array}{l}\text { Centro de Apoio Educacional } \\
\text { e Psicológico (Caep), } 1990 .\end{array}$ & $\begin{array}{l}\text { Psicoterapia } \\
\text { breve, orientação } \\
\text { psicopedagógica, } \\
\text { orientação profissional, } \\
\text { grupos temáticos e de } \\
\text { reflexão, orientação } \\
\text { familiar, orientação a } \\
\text { professores, orientação } \\
\text { e encaminhamentos, } \\
\text { assessoria pedagógica } \\
\text { a docentes e assessoria } \\
\text { pedagógica à Comissão de } \\
\text { Graduação. }\end{array}$ & $\begin{array}{l}\text { Estudantes de graduação } \\
\text { do curso de Medicina da } \\
\text { Faculdade de Medicina de } \\
\text { Ribeirão Preto. }\end{array}$ & $\begin{array}{l}\text { Psicólogo, } \\
\text { pedagogo } \\
\text { e técnico } \\
\text { acadêmico } \\
\text { para apoio } \\
\text { administrativo. }\end{array}$ \\
\hline
\end{tabular}


Quadro 2. (Continuação) Principais dados coletados.

\begin{tabular}{lcccc}
\hline $\begin{array}{c}\text { Autor(es)/ano } \\
\text { de publicação }\end{array}$ & $\begin{array}{c}\text { Formato/ } \\
\text { desenho } \\
\text { metodológico }\end{array}$ & $\begin{array}{c}\text { Nome do serviço/ano de } \\
\text { criação }\end{array}$ & $\begin{array}{c}\text { Intervenções oferecidas } \\
\text { Público-alvo }\end{array}$ & $\begin{array}{c}\text { Profissionais } \\
\text { que compõem o } \\
\text { serviço }\end{array}$ \\
\hline
\end{tabular}

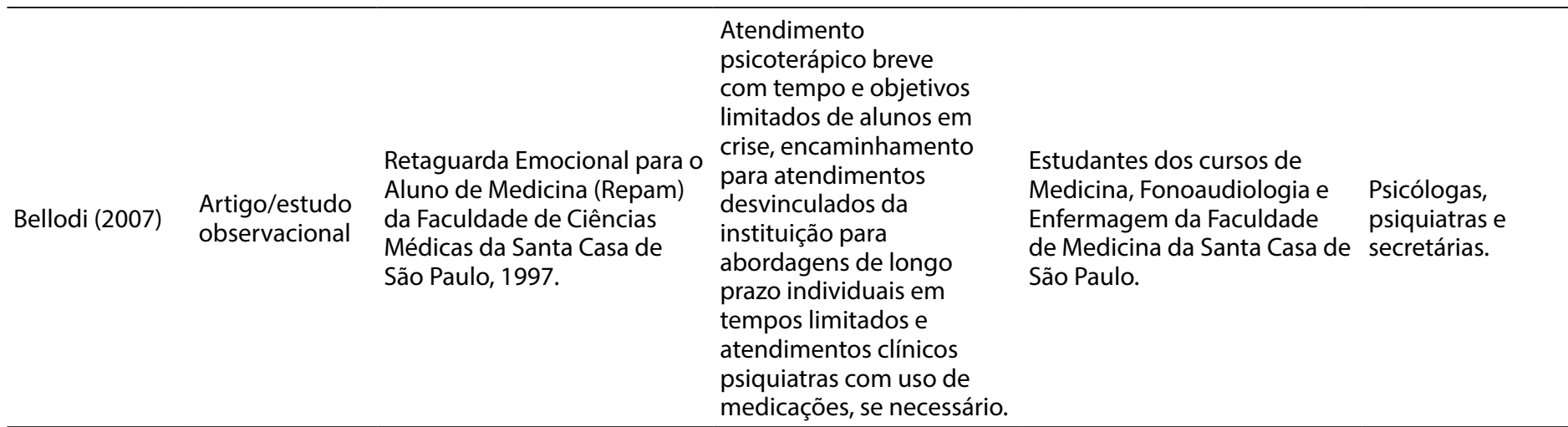

\begin{tabular}{|c|c|c|c|c|c|}
\hline $\begin{array}{l}\text { Millan e Arruda } \\
\text { (2008) }\end{array}$ & $\begin{array}{l}\text { Artigo/ } \\
\text { descrição } \\
\text { histórica }\end{array}$ & $\begin{array}{l}\text { Grupo de Assistência } \\
\text { Psicológica ao Aluno (Grapal) } \\
\text { da Faculdade de Medicina da } \\
\text { Universidade de São Paulo, } \\
\text { criado em } 1983 \text { e iniciado em } \\
1986 .\end{array}$ & $\begin{array}{l}\text { Assistência psicológica e } \\
\text { psiquiátrica ao estudante, } \\
\text { breve entrevista } \\
\text { psicológica de caráter } \\
\text { preventivo com alunos } \\
\text { do primeiro ano do curso, } \\
\text { organização de eventos } \\
\text { para o debate sobre } \\
\text { os serviços de saúde } \\
\text { mental para estudantes e } \\
\text { orientações à família de } \\
\text { estudantes atendidos. }\end{array}$ & $\begin{array}{l}\text { Estudantes de Medicina da } \\
\text { Faculdade de Medicina da } \\
\text { Universidade de São Paulo. }\end{array}$ & $\begin{array}{l}\text { Psicólogos, } \\
\text { psiquiatras, } \\
\text { secretário e } \\
\text { coordenador. }\end{array}$ \\
\hline $\begin{array}{l}\text { De Marco } \\
\text { (2009) }\end{array}$ & Artigo/ensaio & $\begin{array}{l}\text { Grupo de Assistência } \\
\text { Psicológica ao Aluno (Grapal) } \\
\text { da Faculdade de Medicina da } \\
\text { Universidade de São Paulo; } \\
\text { não é informado o ano de } \\
\text { criação. }\end{array}$ & $\begin{array}{l}\text { Entrevista aberta de } \\
\text { apresentação do serviço } \\
\text { para os alunos do primeiro } \\
\text { ano do curso e suporte } \\
\text { psicopedagógico. }\end{array}$ & $\begin{array}{l}\text { Estudantes de Medicina da } \\
\text { Faculdade de Medicina da } \\
\text { Universidade de São Paulo. }\end{array}$ & $\begin{array}{l}\text { Não há essa } \\
\text { informação no } \\
\text { artigo. }\end{array}$ \\
\hline Oliveira (2009) & $\begin{array}{l}\text { Dissertação/ } \\
\text { pesquisa } \\
\text { documental }\end{array}$ & $\begin{array}{l}\text { Serviço de Assistência } \\
\text { Psicológica e Psiquiátrica ao } \\
\text { Estudante na Universidade } \\
\text { Estadual de Campinas } \\
\text { (Sappe/Unicamp), } 1987 .\end{array}$ & $\begin{array}{l}\text { Psicoterapia breve de } \\
\text { orientação psicanalítica, } \\
\text { desenvolvimento de } \\
\text { programas que facilitam a } \\
\text { integração do estudante } \\
\text { no contexto universitário, } \\
\text { pronto atendimento } \\
\text { psicológico (PAP) e } \\
\text { atendimento clínico } \\
\text { psiquiátrico. }\end{array}$ & $\begin{array}{l}\text { Estudantes dos cursos } \\
\text { (graduação e pós-graduação) } \\
\text { oferecidos pela instituição de } \\
\text { ensino superior (Unicamp). }\end{array}$ & $\begin{array}{l}\text { Psicólogos e } \\
\text { psiquiatras. }\end{array}$ \\
\hline $\begin{array}{l}\text { Leão et al. } \\
\text { (2011) }\end{array}$ & $\begin{array}{l}\text { Artigo/estudo } \\
\text { observacional }\end{array}$ & $\begin{array}{l}\text { Mental Health Service (MHS) } \\
\text { e Mentoring Program; não é } \\
\text { informado os anos de criação } \\
\text { dos serviços. }\end{array}$ & $\begin{array}{l}\text { Atendimento e } \\
\text { acompanhamento } \\
\text { psicológicos e programa } \\
\text { de mentoria. }\end{array}$ & $\begin{array}{l}\text { Estudantes de Medicina da } \\
\text { instituição de ensino superior } \\
\text { (FMUSP). }\end{array}$ & $\begin{array}{l}\text { O serviço de } \\
\text { saúde mental } \\
\text { é constituídos } \\
\text { por psicólogos } \\
\text { e psiquiatras, e } \\
\text { o programa de } \\
\text { mentoria conta } \\
\text { com docentes } \\
\text { voluntários que } \\
\text { atuam como } \\
\text { mentores. }\end{array}$ \\
\hline Souza (2011) & $\begin{array}{l}\text { Dissertação/ } \\
\text { estudo } \\
\text { observacional }\end{array}$ & $\begin{array}{l}\text { O nome do serviço ofertado } \\
\text { não foi informado; o ano de } \\
\text { criação foi } 1993 \text { e o ano de } \\
\text { implantação } 1995 .\end{array}$ & $\begin{array}{l}\text { Atendimento psicológico e } \\
\text { psiquiátrico, serviço social, } \\
\text { propostas pedagógicas e } \\
\text { grupos terapêuticos com } \\
\text { assuntos predefinidos. }\end{array}$ & $\begin{array}{l}\text { Estudantes dos cursos } \\
\text { oferecidos pela instituição } \\
\text { e servidores (técnico } \\
\text { administrativos e docentes). }\end{array}$ & $\begin{array}{l}\text { Psicólogos, } \\
\text { psiquiatras } \\
\text { e assistentes } \\
\text { sociais. }\end{array}$ \\
\hline
\end{tabular}


Quadro 2. (Continuação) Principais dados coletados.

\begin{tabular}{|c|c|c|c|c|c|}
\hline $\begin{array}{l}\text { Autor(es)/ano } \\
\text { de publicação }\end{array}$ & $\begin{array}{c}\text { Formato/ } \\
\text { desenho } \\
\text { metodológico }\end{array}$ & $\begin{array}{l}\text { Nome do serviço/ano de } \\
\text { criação }\end{array}$ & Intervenções oferecidas & Público-alvo & $\begin{array}{c}\text { Profissionais } \\
\text { que compõem o } \\
\text { serviço }\end{array}$ \\
\hline Campos (2016) & $\begin{array}{l}\text { Dissertação/ } \\
\text { pesquisa } \\
\text { documental }\end{array}$ & $\begin{array}{l}\text { Serviço de Assistência } \\
\text { Psicológica e Psiquiátrica ao } \\
\text { Estudante na Universidade } \\
\text { Estadual de Campinas } \\
\text { (Sappe/Unicamp), } 1987 .\end{array}$ & $\begin{array}{l}\text { Consultas individuais } \\
\text { de acolhimento e } \\
\text { triagem, intervenções } \\
\text { psicoterápicas breves } \\
\text { de orientação analítica, } \\
\text { associação com redes } \\
\text { e serviços internos e } \\
\text { externos à faculdade, } \\
\text { atendimentos psicológicos } \\
\text { individuais, grupais } \\
\text { e relacionais, pronto } \\
\text { atendimento psicológico } \\
\text { (PAP) e acompanhamentos } \\
\text { psiquiátricos de urgência. }\end{array}$ & $\begin{array}{l}\text { Estudantes de Medicina, } \\
\text { Enfermagem e } \\
\text { Fonoaudiologia (graduação, } \\
\text { pós-graduação e residência), } \\
\text { docentes e funcionários de } \\
\text { diferentes áreas da Unicamp. }\end{array}$ & $\begin{array}{l}\text { Psiquiatras e } \\
\text { psicólogos. }\end{array}$ \\
\hline Pinho (2016) & $\begin{array}{l}\text { Artigo/ } \\
\text { pesquisa } \\
\text { documental }\end{array}$ & $\begin{array}{l}\text { Programa de Atendimento } \\
\text { Psicológico do Meio Oeste } \\
\text { Catarinense, } 2004 .\end{array}$ & $\begin{array}{l}\text { Atendimento psicológico } \\
\text { de abordagem cognitivo- } \\
\text { comportamental e } \\
\text { encaminhamento de } \\
\text { estudantes para tratamento } \\
\text { com psicoterapia de apoio } \\
\text { desvinculado da instituição. }\end{array}$ & $\begin{array}{l}\text { No ano de sua implantação, } \\
\text { atendia apenas estudantes de } \\
\text { Medicina. Em 2005, começou } \\
\text { a ser ofertado a todos os } \\
\text { estudantes dos demais cursos } \\
\text { oferecidos pela instituição. }\end{array}$ & $\begin{array}{l}\text { Não há essa } \\
\text { informação no } \\
\text { artigo. }\end{array}$ \\
\hline
\end{tabular}

\section{Acolhimento aberto com} horários de funcionamento preestabelecidos e
Programa de Atenção em Saúde Mental para

Malajovich et Artigo/reflexão al. (2017) teórico-clínica
Estudantes Universitários (Proasme); não consta o ano de criação do programa.
Murakami et al. Artigo/relato (2018) de experiência

Centro de Apoio Educacional e Psicológico (Caep), 1990. entrevistas individuais que visam à realização de um diagnóstico situacional; as propostas terapêuticas são diversas e formuladas a partir do contato com o estudante.
Estudantes dos cursos oferecidos pela instituição de ensino superior (não informada no artigo analisado).
Psicólogos e psiquiatras que realizam interconsultas.
Triagem psicológica, psicoterapia breve, orientação educacional (psicopedagógica e/ ou pedagógica), aconselhamento psicológico, programa

de tutoria na categoria mentoring, oficinas de orientações psicopedagógica e pedagógica, grupos de reflexão e grupos focais, e prestação de consultoria à Comissão de Graduação e aos docentes da instituição.
Na época da criação, atendia apenas estudantes de Medicina, sendo expandido, posteriormente, para os cursos de Fisioterapia, Fonoaudiologia, Nutrição, Terapia Ocupacional, Informática Biomédica e Ciência Biomédicas.
Três

psicólogos, um psicopedagogo, um pedagogo e um técnico administrativo.
Atendimento psicológico psicoterápico breve baseado em consultas individuais; oferta de estágio profissional na área de psicologia a partir de 2019.
Espaço de Atenção

Neves et al. Artigo/relato Psicossocial (Epsico) (2019) de experiência idealizado e proposto 2015, e efetivado em 2018
Na época de sua criação, contava com cinco psicólogos

Estudantes dos cursos oferecidos pela instituição de e um terapeuta ensino superior. ocupacional.

Atualmente é constituído por três psicólogos. 
Quadro 2. (Continuação) Principais dados coletados.

\begin{tabular}{|c|c|c|c|c|c|}
\hline $\begin{array}{l}\text { Autor(es)/ano } \\
\text { de publicação }\end{array}$ & $\begin{array}{c}\text { Formato/ } \\
\text { desenho } \\
\text { metodológico }\end{array}$ & $\begin{array}{l}\text { Nome do serviço/ano de } \\
\text { criação }\end{array}$ & Intervenções oferecidas & Público-alvo & $\begin{array}{c}\text { Profissionais } \\
\text { que compõem o } \\
\text { serviço }\end{array}$ \\
\hline Mendes (2019) & $\begin{array}{l}\text { Artigo/ } \\
\text { descrição } \\
\text { histórica }\end{array}$ & $\begin{array}{c}\text { Assistência Psicológica aos } \\
\text { Alunos (APP) da Pontifícia } \\
\text { Universidade Católica de } \\
\text { Minas Gerais; serviço criado } \\
\text { em 2002. }\end{array}$ & $\begin{array}{c}\text { Acolhida psicossocial do } \\
\text { estudantes realizada por } \\
\text { meio de escutas pontuais, } \\
\text { atendimento psicoterápico } \\
\text { breve em casos de } \\
\text { urgência subjetiva e } \\
\text { encaminhamento a } \\
\text { atendimento psicológico } \\
\text { desvinculado da instituição } \\
\text { para acompanhamentos } \\
\text { longitudinais. }\end{array}$ & Estudantes da instituição. & $\begin{array}{l}\text { Psicólogos, } \\
\text { monitores e } \\
\text { estagiários de } \\
\text { psicologia. }\end{array}$ \\
\hline $\begin{array}{l}\text { Ribeiro et al. } \\
\text { (2019) }\end{array}$ & $\begin{array}{c}\text { Artigo/ } \\
\text { pesquisa } \\
\text { documental }\end{array}$ & $\begin{array}{l}\text { Núcleo de Apoio } \\
\text { Psicopedagógico ao } \\
\text { Estudante (Napem) da } \\
\text { Faculdade de Medicina da } \\
\text { Universidade Federal de } \\
\text { Minas Gerais, } 2004 .\end{array}$ & $\begin{array}{c}\text { Intervenções psicológicas } \\
\text { a partir do preenchimento } \\
\text { de uma ficha de inscrição } \\
\text { pelo aluno. }\end{array}$ & $\begin{array}{l}\text { Estudantes da Faculdade de } \\
\text { Medicina da Universidade } \\
\text { Federal de Minas Gerais } \\
\text { matriculados nos cursos de } \\
\text { Medicina, Fonoaudiologia e } \\
\text { Tecnologia em Radiologia. }\end{array}$ & $\begin{array}{l}\text { Não há essa } \\
\text { informação no } \\
\text { artigo. }\end{array}$ \\
\hline
\end{tabular}

\section{Público-alvo}

Todos os serviços de apoio à saúde mental descritos nos estudos selecionados atendem estudantes de Medicina. No entanto, alguns desses serviços são disponibilizados também para graduandos de outros cursos.

De forma geral, podemos observar que a maioria dos serviços de apoio foi implementada com o objetivo de prestar assistência psicológica e psicopedagógica apenas aos estudantes de Medicina, possivelmente por causa da maior prevalência de transtornos mentais nesse grupo, conforme evidenciado por Pacheco et al. ${ }^{2}$. Como exemplo, temos o serviço de apoio ao estudante da Universidade do Oeste de Santa Catarina (Unoesc), implantado em 2004 para atendimento de discentes do curso de Medicina²1.

Com a ampliação do serviço e/ou a implantação de outros cursos nas instituições, alguns serviços assistenciais em saúde mental passaram a receber também estudantes provenientes de outros cursos de graduação, como é o caso do Caep da FMRP que, em 2002, começou a atender às demandas de outros cursos da área da saúde, como Fisioterapia, Fonoaudiologia e Nutrição ${ }^{15}$. Em apenas um dos estudos incluídos, o serviço abordado também atendia discentes regularmente matriculados na pós-graduação da instituição ${ }^{12}$.

\section{Profissionais envolvidos}

Os serviços de apoio à saúde mental são compostos, na maioria dos casos, por equipes multiprofissionais que variam em número e categorias profissionais envolvidas.

Os serviços que oferecem atendimento psicológico e psiquiátrico são comumente compostos por psicólogos e psiquiatras. As equipes podem incluir também psicopedagogos, pedagogos, técnicos administrativos, secretários e terapeutas ocupacionais. O APP da PUC Minas ${ }^{23}$ e o Serviço de Psicoterapia Analítica do Ambulatório de Clínica Psiquiátrica do Hospital das Clínicas de Ribeirão Preto $^{29}$ contam também com estagiários provenientes do curso de graduação em Psicologia e médicos residentes em psiquiatria, respectivamente.

Já os programas de tutoria ou mentoring oferecidos pelas Faculdades de Medicina da USP ${ }^{19}$ e da UFMG ${ }^{20}$ são compostos pelos próprios docentes do curso de graduação, geralmente em caráter voluntário.

Em três dos estudos incluídos, não há informações sobre os profissionais que compõem o serviço assistencial ${ }^{21,26,27}$.

É importante ressaltar que alguns serviços apresentam uma instabilidade quanto ao número de profissionais. No estudo em que o Epsico da UEA é descrito, relata-se que, em 2015, ano da elaboração do projeto, o serviço contava com cinco psicólogas e uma terapeuta ocupacional. Contudo, na data de elaboração do artigo, em 2019, somente três psicólogas atuavam no serviço ${ }^{16}$.

Pode-se supor que a ausência de um quadro fixo de profissionais seja um fator que dificulte o desenvolvimento de projetos de longo prazo, assim como a consolidação e ampliação dos serviços de apoio à saúde mental dentro das instituições de ensino superior do Brasil.

\section{CONSIDERAÇÕES FINAIS}

Os pontos fortes desta revisão foram a busca sistemática e abrangente na literatura, e as lacunas de conhecimento encontradas a partir das informações coletadas dos estudos incluídos.

Diante dos resultados apresentados, observou-se que os serviços de apoio à saúde mental abordados pelos estudos 
têm o objetivo comum de promoção da saúde mental e, assim, da qualidade de vida do estudante de Medicina. Diferentes modalidades de intervenção, por meio de encontros individuais e/ou coletivos, visam acolher e apoiar o estudante em suas demandas acadêmicas, psicológicas e sociais.

A maioria dos serviços de apoio disponibiliza um primeiro acolhimento ao estudante, configurando-se como espaço de escuta, e intervenções de curto prazo, com predomínio da psicoterapia breve. O acesso a esses serviços depende, em geral, da iniciativa do próprio universitário em procurá-los, diante de alguma intercorrência que resulte em prejuízos à sua saúde mental.

Todavia, foram encontradas algumas limitações na realização do estudo. As publicações sobre os serviços de apoio ao estudante são escassas quando comparadas ao número de instituições que oferecem o curso de graduação em Medicina no Brasil. Além disso, os estudos têm caráter mais descritivo, quando o ideal seria haver, além da descrição, uma avaliação das intervenções oferecidas pelos serviços de apoio, o que teria mais relevância que os relatos de experiência.

As publicações disponíveis foram realizadas, em sua maioria, por centros de ensino superior públicos da Região Sudeste, o que dificulta o mapeamento e a identificação de informações a respeito de serviços de apoio ofertados por instituições de outras regiões do país.

O predomínio de estudos que relatam serviços de apoio em instituições de ensino superior públicas pode sugerir viés de publicação. É possível que outros centros de ensino privados possuam serviços destinados a seus estudantes, mas que, por motivos não identificados nesta pesquisa, não publiquem sobre o tema.

É importante destacar ainda que os estudos não apresentam o perfil socioeconômico dos estudantes assistidos pelos serviços de apoio, nem há menção quanto à presença de alunos que ingressaram por cotas ou que são apoiados pelas políticas de permanência. Conhecer tal perfil seria importante para a elaboração de estratégias de assistência aos estudantes, sobretudo para garantir ao grupo economicamente vulnerável o devido seguimento de seu tratamento.

Outra limitação é o fato de a maioria dos estudos incluídos nesta revisão não detalhar o modo de implementação dos serviços ou como as intervenções propostas são colocadas em prática. Também não foram encontradas informações sobre possíveis dificuldades em se manter uma equipe multiprofissional ou sobre o impacto das intervenções na saúde mental dos estudantes contemplados.

Além disso, é válido reconhecer que muitas outras ações voltadas para a promoção da saúde mental são desenvolvidas no ambiente acadêmico por frentes de saúde mental, ligas e outros setores desvinculados dos serviços de apoio, e, dessa forma, não fizeram parte desta revisão.

Quanto à relação das instituições de ensino com os serviços de apoio, podemos perceber que, apesar do seu papel decisivo no processo de criação desses serviços, não há informações nos estudos sobre a participação da gestão acadêmica em outros momentos. Não foi abordado, por exemplo, se os centros de ensino desenvolvem ações regulares de promoção de saúde mental no contexto acadêmico ou se há a preocupação de efetuar mudanças curriculares e organizacionais visando atender às demandas de saúde mental.

Por último, apesar de o objetivo principal desta revisão ter sido mapear, por meio de publicações, os serviços de apoio à saúde mental existentes nas instituições de ensino superior do Brasil, não podemos inferir que os serviços descritos nos estudos incluídos ainda estão ativos. É possível, ainda, que tenham ocorrido mudanças, tanto no funcionamento como na composição dos serviços.

Tais lacunas apontam para a necessidade de mais pesquisas sobre atenção à saúde mental do estudante de Medicina, assim como de maior incentivo institucional para que projetos e estudos com esse foco sejam desenvolvidos. Espera-se que, mediante mais pesquisas sobre essa temática, as discussões sobre saúde mental possam ser ampliadas e estratégias comprovadamente efetivas sejam implementadas nos serviços de apoio aos estudantes, a fim de expandir e consolidar a ação desses serviços dentro das instituições de ensino superior do Brasil.

\section{CONTRIBUIÇÃO DAS AUTORAS}

Todas as autoras participaram do desenho do estudo, da construção do protocolo da revisão, das estratégias de busca, da análise e interpretação dos dados coletados, da redação do artigo, da revisão crítica do conteúdo intelectual e da aprovação da versão final a ser publicada.

\section{CONFLITO DE INTERESSES}

As autoras declaram não haver conflito de interesses neste estudo.

\section{FINANCIAMENTO}

Esta pesquisa foi apoiada pelo Programa Institucional de Bolsas de Iniciação Científica do Conselho Nacional de Desenvolvimento Científico e Tecnológico (Pibic/CNPq) e pelo Programa de Iniciação Científica da Escola Superior de Ciências da Saúde (PIC/ESCS). 


\section{REFERENCIAS}

1. do Amaral GF, Gomide LMP, Batista MP, Píccolo PP, Teles TBG, de Oliveira PM, et al. Sintomas depressivos em acadêmicos de medicina da Universidade Federal de Goiás: um estudo de prevalência. Rev Psiquiatr. Rio Gd Sul. 2008;30(2):124-30 [access in 24 jun 2020]. Available from: https://www. scielo.br/scielo.php?pid=S0101-81082008000300008\&script $=$ sci_ abstract\&tIng=pt.

2. Pacheco JP, Giacomin HT, Tam WW, Ribeiro TB, Arab C, Bezerra IM, et al. Mental health problems among medical students in Brazil: a systematic review and meta-analysis. Rev Bras Psiquiatr. 2017;39(4):369-78 [access in 24 jul 2020]. Available from: https://www.scielo.br/scielo.php?script=sci_ arttext\&pid=S1516-44462017000400369.

3. Quek TTC, Tam WWS, Tran BX, Zhang M, Zhang Z, Ho CSH, et al. The Global Prevalence of Anxiety Among Medical Students: A meta-analysis. Int J Environ Res Public Health. 2019 Jul 31;16(15):2735 [access in 27 jul 2020]. Available from: https://www.ncbi.nlm.nih.gov/pmc/articles/ PMC6696211/.

4. Tenório LP, Argolo VA, As HP, Melo EV, Costa EFO. Saúde mental de estudantes de escolas médicas com diferentes modelos de ensino. Rev Bras Educ Med. 2016;40(4):574-82. doi: 10.1590/1981-52712015v40n $4 \mathrm{e} 00192015$.

5. Cerchiari EAN, Caetano D, Faccenda O. Utilização do serviço de saúde mental em uma universidade pública. Psicol Ciênc Prof. 2005;25(2):25265 [access in 10 jul 2020]. Available from: https://www.scielo.br/scielo. php?pid=S1414-98932005000200008\&script=sci_abstract\&tlng=pt.

6. de Assis $A D$, de Oliveira AGB. Vida universitária e saúde mental: atendimento às demandas de saúde e saúde mental de estudantes de uma universidade brasileira. Cadernos Brasileiros de Saúde Mental. 2010;2:159-77 [access in 31 jul 2020]. Available from: https://periodicos. ufsc.br/index.php/cbsm/article/view/68464/0.

7. Millan LR, De Marco OLN, Rossi E, de Arruda PCV. O universo psicológico do futuro médico: vocação, vicissitudes e perspectivas. São Paulo: Casa do Psicólogo; 1999.

8. Baldassin SP, Espin Neto J, Dagostino SB, Calado TBM, Guimarães KBS, Colares MFA, et al. I Fórum Paulista de Serviços de Apoio ao Estudante de Medicina - Forsa Paulista - "A carta de Marília". Rev Bras Educ Med. 2016;40(4):537-9. doi: 10.1590/1981-52712015v40n4e00862016.

9. Nogueira JC. Saúde mental em estudantes do ensino superior: fatores protetores e fatores de vulnerabilidade [tese]. Lisboa: Universidade de Lisboa; 2017.

10. Elsevier. Mendeley - Reference Management Software and Researcher Network. Version 1803 [access in 18 sep 2020]. Available from: www. mendeley.com.

11. Veritas Health Innovation. Covidence systematic review software. Melbourne, Australia [access in 20 sep 2020]. Available from: www. covidence.org.

12. Campos, CRF. Perfil sociodemográfico, clínico e acadêmico de estudantes universitários que passaram por atendimento psiquiátrico no Serviço de Assistência Psicológica e Psiquiátrica ao Estudante da Universidade Estadual de Campinas (Sappe-Unicamp) entre 2004 e 2011 [dissertação]. Campinas: Universidade Estadual de Campinas; 2016 [access in 10 jul 2020]. Available from: http://repositorio.unicamp.br/jspui/bitstream/ REPOSIP/321049/1/Campos_ClaudiaRibeiroFranulovic_M.pdf.

13. de Souza GG. Atenção psicológica em universidade: a experiência de estudantes como clientes [dissertação]. Campinas: Pontifícia Universidade Católica de Campinas; 2011 [access in 10 jul 2020]. Available from: http://tede.bibliotecadigital.puccampinas.edu.br:8080/jspui/bitstream/ tede/271/1/Grasiela\%20Gomide\%20de\%20Souza.pdf.

14. Oliveira MLC. Caracterização sociodemográfica, acadêmica e clínica dos estudantes atendidos no Serviço de Assistência Psicológica e Psiquiátrica ao Estudante (Sappe) de 1987 a 2004 [dissertação]. Campinas: Universidade Estadual de Campinas; 2009 [access in 10 jul 2020]. Available from: http://repositorio.unicamp.br/jspui/handle/REPOSIP/308732.
15. Murakami K, Barros GC, Peres CM, Flauzino RH, Colares MFA. Atuações de um centro educacional e psicológico junto a estudantes universitários. Rev Bras Orientac Prof. 2018;19:109-19 [access in 10 jul 2020]. Available from: http://pepsic.bvsalud.org/scielo.php?script=sci_arttext\&pid=S167933902018000100012\&lng=pt\&nrm=iso.

16. das Neves ALM, Ramos ES, Marangoni VL, Martins GC. Saúde mental e universidade: experiência do "Espaço de Atendimento Psicossocial" (Epsico) Trab En(Cena). 2019;4(2):531-42 [access in 31 jul 2020]. Available from: https:// sistemas.uft.edu.br/periodicos/index.php/encena/article/view/7482.

17. Cianflone ARL, Figueiredo JFC, Colares MFA. O Centro de Apoio Educacional e Psicológico (Caep) da Faculdade de Medicina de Ribeirão Preto (USP): história e perspectivas. Medicina (Ribeirão Preto). 2002;35(3):392-6 [access in 31 jul 2020]. Available from: http://www.revistas.usp.br/rmrp/ article/view/857/869.

18. Millan LR, de Arruda PCV. Assistência psicológica ao estudante de medicina: 21 anos de experiência. Rev Assoc Med Bras. 2008;54(1):904 [access in 31 jul 2020]. Available from: https://www.scielo.br/scielo. php?script=sci_arttext\&pid=S0104-42302008000100027.

19. Leão PBOS, Martins LAN, Menezes PR, Bellodi PL. Well-being and help-seeking: an exploratory study among final-year medical students. Rev Assoc Med Bras. 2011;4(57):379-86 [access in 10 aug 2020]. Disponíve em: https://www.scielo.br/scielo.php?script=sci_ arttext\&pid=S0104-42302011000400009.

20. Martins AF, Bellodi PL. Mentoring: uma vivência de humanização e desenvolvimento no curso médico. Interface (Botucatu). 2016;20(58):71526 [access in $1^{\circ}$ aug 2020];20(58):715-26. Available from: https://www. scielo.br/scielo.php?pid=S141432832016000300715\&script=sci abstract\&tlng=pt.

21. Pinho R. Caracterização da clientela de um programa de atendimento psicológico a estudantes universitários. Psicol Conoc Soc. 2016;6(1):114 30 [access in $1^{\circ}$ aug 2020]. Available from: http://www.scielo.edu.uy/ scielo.php?script=sci_arttext\&pid=S1688-70262016000100006.

22. Brasil. Sistema e-MEC. Brasília: Ministério da Educação; 2020 [access in 16 aug 2020]. Available from: https://emec.mec.gov.br.

23. Mendes AA. A saúde mental de jovens universitários: apontamentos sobre a parceria de trabalho entre a APP-PUC Minas e o BAPU de Rennes na França. Pretextos - Revista da Gradução em Psicologia da PUC Minas. 2019;4(7):50-60 [access in 2 aug 2020]. Disponíve em: http://periodicos. pucminas.br/index.php/pretextos/article/view/20750/15015.

24. Bellodi PL. Retaguarda Emocional para o Aluno de Medicina da Santa Casa de São Paulo (Repam): realizações e reflexões. Rev Bras Educ Med. 2007;31:5-14 [access in 2 aug 2020]. Available from: https://www.scielo.br/ scielo.php?pid=S0100-55022007000100002\&script=sci_abstract\&tlng=pt.

25. Hilu L, Gisi ML. Produção científica no Brasil: um comparativo entre as universidades públicas e privadas. X Congresso Nacional de Educação Educere; 2011. Curitiba: PUCPR; 2011. p. 7-10 [access in 2 aug 2020]. Available from: https://educere.bruc.com.br/arquivo/pdf2011/5221_3061.pdf.

26. De Marco OLC. O estudante de medicina e a procura de ajuda. Rev Bras Educ Med. 2009;33(3):476-81 [access in 2 aug 2020]. Available from: https://www.scielo.br/pdf/rbem/v33n3/19.pdf.

27. Ribeiro MMF, Melo JDC, Rocha AMC. Avaliação da demanda preliminar de atendimento dirigida pelo aluno ao Núcleo de Apoio Psicopedagógico ao Estudante da Faculdade de Medicina (Napem) da Universidade Federal de Minas Gerais. Rev Bras Educ Med. 2019;43:91-7 [access in $1^{\circ}$ aug 2020]. Available from: https://www.scielo.br/scielo.php?pid=S0100 $55022019000500091 \&$ script=sci_arttext.

28. Malajovich N, Vilanova A, Frederico C, Cavalcanti MT, Velasco LB. Ajuventude universitária na contemporaneidade: a construção de um serviço de atenção em saúde mental para estudantes. Mental. 2017;11(21):356-77 [access in 3 aug 2020]. Available from: http://pepsic.bvsalud.org/scielo. php?script=sci_arttext\&pid=S1679-44272017000200005.

29. Fernandez JM, Rodrigues CRC. Estudo retrospectivo de uma população de estudantes de medicina atendidos no ambulatório de clínica psiquiátrica do Hospital das Clínicas da Faculdade de Medicina de Ribeirão Preto. Medicina (Ribeirão Preto). 1993;6(2):258-69 [access in $1^{\circ}$ aug 2020]. Available from: https://pesquisa.bvsalud.org/portal/resource/pt/lil-128945.

This is an Open Access article distributed under the terms of the Creative Commons Attribution License, which permits unrestricted use, distribution, and reproduction in any medium, provided the original work is properly cited. 